\title{
Hybridization and yarns configuration effects on flexural dynamic and static properties of pultruded hybrid kenaf/glass fiber composites
}

\begin{abstract}
ABSTRCT
Six different hybrid composites with various hybrid fiber mixing ratio were fabricated using the pultrusion technique. The effect of fibers hybridization and fibers configuration with respect to the loading axis were studied on flexural dynamic and static properties. In low fiber mixing ratio of about 0.09 , the radial configuration of kenaf yarns in the core region had no significant effect on dynamic flexural modulus. The radial configuration of the kenaf yarns in the core of the composites and bilateral configuration of the glass yarns in the shell region showed a considerable effect on damping factor. The bilateral configuration of glass yarns in the shell region indicated a noticeable effect on the static flexural modulus, strength and the strain at break. The regression analysis showed a significant relationship between the static and dynamic modulus, and the dynamic flexural modulus explained $98 \%$ of the variability of the static flexural modulus.
\end{abstract}

Keyword: Hybrid; Natural fibers; Mechanical properties; Pultrusion 